\title{
Optical clock technologies for global navigation satellite systems
}

\author{
Thilo Schuldt ${ }^{1}$ (1) $\cdot$ Martin Gohlke ${ }^{1}$ Markus Oswald ${ }^{1,2} \cdot$ Jan Wüst ${ }^{1} \cdot$ Tim Blomberg $^{1} \cdot$ Klaus Döringshoff $^{3,4}$. \\ Ahmad Bawamia $^{3}$. Andreas Wicht ${ }^{3} \cdot$ Matthias Lezius $^{5} \cdot$ Kai Voss $^{6} \cdot$ Markus Krutzik $^{3,4}$. Sven Herrmann ${ }^{2}$. \\ Evgeny Kovalchuk $^{3,4}$. Achim Peters ${ }^{3,4}$. Claus Braxmaier ${ }^{1,2}$
}

Received: 5 October 2020 / Accepted: 3 March 2021 / Published online: 5 April 2021

(c) The Author(s) 2021

\begin{abstract}
Future generations of global navigation satellite systems (GNSSs) can benefit from optical technologies. Especially optical clocks could back-up or replace the currently used microwave clocks, having the potential to improve GNSS position determination enabled by their lower frequency instabilities. Furthermore, optical clock technologies-in combination with optical inter-satellite links—enable new GNSS architectures, e.g., by synchronization of distant optical frequency references within the constellation using time and frequency transfer techniques. Optical frequency references based on Doppler-free spectroscopy of molecular iodine are seen as a promising candidate for a future GNSS optical clock. Compact and ruggedized setups have been developed, showing frequency instabilities at the $10^{-15}$ level for averaging times between $1 \mathrm{~s}$ and $10,000 \mathrm{~s}$. We introduce optical clock technologies for applications in future GNSS and present the current status of our developments of iodine-based optical frequency references.
\end{abstract}

Keywords Optical clock $\cdot$ Iodine reference $\cdot$ Space instrumentation $\cdot$ Future GNSS

\section{Introduction}

Over the last decades, optical clock technologies evolved, recently demonstrating frequency instabilities at the $10^{-18}$ level for integration times of a few thousand seconds (Ushijima et al. 2015; McGrew et al. 2018). While becoming more and more widespread technology in and outside laboratories on Earth, also space applications_including GNSS — can benefit from the recent advancement of optical technologies. Optical clocks surpass the performance of the currently used GNSS microwave clocks by several orders of magnitude.

Thilo Schuldt

Thilo.schuldt@dlr.de

1 German Aerospace Center (DLR), Institute of Space Systems, Bremen, Germany

2 Center of Applied Space Technology and Microgravity, University of Bremen, Bremen, Germany

3 Ferdinand-Braun-Institut gGmbH, Leibniz-Institut Für Höchstfrequenztechnik, Berlin, Germany

4 Institute of Physics, Humboldt-Universität zu Berlin, Berlin, Germany

5 Menlo Systems GmbH, Martinsried, Germany

6 SpaceTech GmbH, Immenstaad, Germany
On the one hand, optical clocks could back-up or replace the currently used microwave clocks (Droz et al. 2006), on the other hand, optical clock technologies-in combination with optical inter-satellite links-enable new GNSS architectures. One example is the proposed Kepler architecture (Giorgi et al. 2019) which foresees 24 satellites in mediumearth orbits (MEO, in three orbital planes, similar to the current Galileo system), together with 6 satellites in low-Earth orbit (LEO). All Kepler satellites carry optical frequency references which are intra-system synchronized using bidirectional optical inter-satellite links together with time and frequency transfer techniques. In the current baseline, the satellites are equipped with frequency references based on optical resonators, providing the required high short-term stability where the round-trip time of the light within one orbital plane (of about $0.1 \mathrm{~s}$ ) is relevant. The LEO satellites additionally carry mid- to long-term stable absolute optical clocks based on Doppler-free spectroscopy of molecular iodine for the definition of the system time.

In the following, we first give a short overview of optical clock technologies for space, together with their current technology development status. We then detail our developments with respect to iodine-based optical frequency references for applications in space and present the mission 
concept COMPASSO, a proposed DLR in-orbit verification mission on the International Space Station (ISS).

Please note that we refer to a "clock" for a system which delivers a countable frequency signal in the radio frequency range, and where a time signal can be derived from, as it is e.g., the case in GNSS applications. When being based on an absolute and known frequency, such a system is often also referred to as a "frequency standard".

\section{Optical clock technologies for space applications}

Optical clock technologies include a variety of different implementations, all having their own assets and drawbacks. Looking at space applications, in addition to the demonstrated performance such as frequency stability and accuracy, it is necessary to consider the size, weight, power consumption, robustness, and reliability of the optical reference.

Ultimate frequency stability at the $10^{-18}$ level is shown by optical lattice and single-ion clocks in rather complex laboratory setups (Ushijima et al. 2015; McGrew et al. 2018; Delehay and Lacroute 2018). Technology development with respect to transportable setups has been initiated (Koller et al. 2017; Cao et al. 2017; Brewer et al. 2019; Hannig et al. 2019), and a compact setup of a ${ }^{88} \mathrm{Sr}$ lattice clock has been realized, where space-related design criteria have been considered (Bongs et al. 2015; Origlia et al. 2018). Such optical clocks require several lasers, a vacuum chamber and a cavity pre-stabilization of the clock laser to achieve their outstanding frequency instability enabled by millihertz linewidth transitions.

Optical atomic beam standards, e.g., using $\mathrm{Ca}$ or $\mathrm{Sr}$ atoms show a lower complexity and can be realized in more compact setups. However, similar to optical lattice and singleion clocks, they require a vacuum chamber and use cavity pre-stabilization of the clock laser. With a compact setup of a Ca beam standard, frequency instabilities of $1.8 \times 10^{-15}$ at an integration time of $1600 \mathrm{~s}$ have been demonstrated (Shang et al. 2017). A compact and ruggedized $\mathrm{Sr}$ beam standard for application on a sounding rocket is currently developed at HU Berlin (Gutsch et al. 2019).

Gas-cell-based optical frequency references have modest complexity without need of a vacuum chamber or prestabilization and can be realized with small dimensions, weight and power budgets. They typically employ modulation transfer spectroscopy (MTS) or frequency modulation spectroscopy (FMS) of optical transitions with linewidths of the order of MHz. With a compact Rubidium-based frequency reference using MTS near $420 \mathrm{~nm}$, frequency instabilities of $2.1 \times 10^{-15}$ at an integration time of $80 \mathrm{~s}$ have been claimed, deduced from the error signal (Zhang et al. 2017; Chang et al. 2019). Iodine-based frequency references near
$532 \mathrm{~nm}$ have been realized for many decades, resulting in compact and ruggedized setups, also with respect to applications in space (Nyholm et al. 2003; Leonhard and Camp 2006; Zang et al. 2007; Argence et al. 2010; Schuldt et al. 2017; Döringshoff et al. 2017), showing frequency instabilities at the $10^{-15}$ level for integration times between 1 and $1000 \mathrm{~s}$. A very compact setup has been successfully flown on a sounding rocket, together with a frequency comb (Schkolnik et al. 2017; Döringshoff et al. 2019). Frequency stabilization to iodine transitions near $515 \mathrm{~nm}$ is investigated within the Japanese proposed space gravitational wave detector DECIGO (Deci-Hertz Interferometer Gravitational Wave Observatory) where a compact setup has been realized (Suemasa et al. 2017) and by a French collaboration using a frequency tripled output at $514 \mathrm{~nm}$ of Telecom laser technology at a wavelength near $1542 \mathrm{~nm}$ (Barbarat et al. 2018).

The $\mathrm{Rb}$ two-photon transition (TPT) at $778 \mathrm{~nm}$ is often used as a frequency standard, providing Doppler-free spectroscopy. No laser pre-stabilization and no vacuum chamber are required. While several setups have been realized in the past, recent developments include a compact setup for applications as a successor to the atomic frequency standard in GPS with demonstrated frequency instabilities at the $10^{-15}$ level (Martin et al. 2018). Furthermore, an integrated $\mathrm{Rb}$ clock has been realized, using a micro-fabricated rubidium gas cell in combination with a microcomb (Newman et al. 2019; Maurice et al. 2020).

Optical clocks require a frequency comb to transfer the stability of the clock laser to a radio frequency and thus to provide a countable clock signal. Developments for space applications are already initiated, and compact frequency combs have been successfully flown on a sounding rocket (Lezius et al. 2016; Döringshoff et al. 2019; Pröbster et al. 2021).

Table 1 summarizes the key figures of the technologies detailed above. For comparison, it also includes the space-grade microwave references currently used on Galileo [rubidium atomic frequency reference (RAFS) and passive hydrogen maser (PHM)] where data are taken from the publicly available data sheets. For the optical references, the given values on performance, i.e., frequency stability, are taken from the corresponding publications, together with the values on size, weight and power (SWaP) budgets, if given. As the technology development status of the technologies is quite different-ranging from transportable and compact setups to implementations dedicated for space- the entries cannot directly be compared. It is e.g., assumed that within a dedicated development, the budgets of lattice and ion clocks can be significantly reduced with respect to the current transportable setups (Takamoto et al. 2020). Also, long-term stability of the optical reference is often not yet investigated. However, the summary in Table 1 can be taken as the basis for necessary trade-offs, e.g., between required 


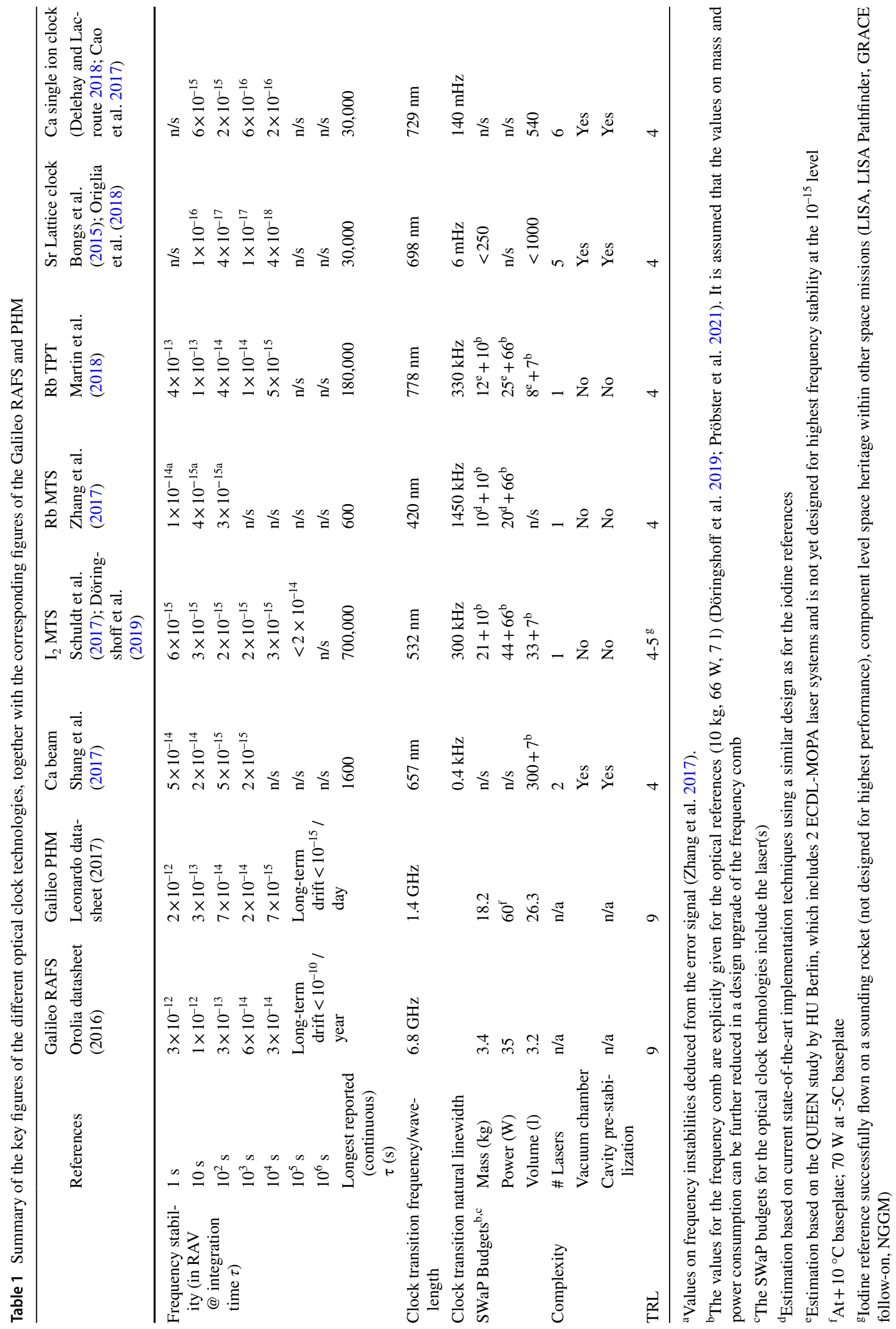


frequency stability, SWaP budgets and robustness/complexity, and also concerning the necessary time frame to develop a space-qualified optical clock.

In Table 1, also the technology maturity is assessed and quantified by the so-called Technology Readiness Level (TRL). The highest level (TRL9) is reached for flight-proven components and systems with demonstrated performance in space operation. The lowest level (TRL1) corresponds to the observation and reporting of basic principles. Functional verification in a typical laboratory environment corresponds to TRL4, the full-scale engineering model with successful environmental testing to TRL6.

At DLR and the University of Bremen, iodine-based frequency references using modulation transfer spectroscopy have been investigated for several years with respect to applications in space, including missions to measure the earth's gravity field (Nicklaus et al. 2017) and space-borne gravitational wave detection (Schuldt et al. 2019). Such references can be realized compact and ruggedized, with small dimensions, mass and power consumption. Typically, the $\mathrm{a}_{10}$ component of the $\mathrm{R}(56) 32-0$ transition in ${ }^{127} \mathrm{I}_{2}$ near a wavelength of $532 \mathrm{~nm}$ is used for frequency stabilization. It is a standard frequency, recommended by the International Bureau of Weights and Measures (Bureau International des Poids et Mesures, BIPM) with a relative standard uncertainty of $8.9 \times 10^{-12}$ (Riehle et al. 2018). As the laser is operated at $1064 \mathrm{~nm}$, iodine-based frequency references can rely on space heritage of the laser and laser components, developed e.g., within the missions LISA (Laser Interferometer Space Antenna), LISA Pathfinder and GRACE (Gravity Recovery and Climate Experiment) follow-on as well as mission concepts developed within the NGGM (Next Generation Gravity Mission) program by the European Space Agency (ESA). Furthermore, commercial laser communication terminals operate at the same wavelength. Iodine-based optical frequency references are seen as a promising candidate for an optical clock for future GNSS showing lower frequency instabilities as the currently used PHM. Relying on an extensive heritage, a flight model fulfilling the Galileo requirements could be realized on short timescale and at moderate costs. As a perspective, this technology is seen as an initiating step toward a routinely applied and reliable optical clock technology in space, also paving the way for future ultra-high performance optical single-ion and lattice clocks in space.

\section{Absolute frequency references based on Doppler-free spectroscopy of molecular iodine near $532 \mathrm{~nm}$}

Molecular iodine offers very strong absorption lines near $532 \mathrm{~nm}$ which can easily be accessed with a laser system at a wavelength of $1064 \mathrm{~nm}$ using second harmonic generation (SHG), also realized in compact and ruggedized setups for applications in space.

Over the last years, DLR - in collaboration with the Humboldt-Universität zu Berlin and the University Bremen-has developed several setups of iodine-based frequency references with a roadmap toward space applications. The optical setups for modulation transfer spectroscopy are realized using an adhesive bonding technology where the optical components are joint to a baseplate made of glass (or glass ceramics, respectively) with a space-qualified two-component epoxy, see Fig. 1. This ensures the high thermal and mechanical stability of the optical system needed for operation in space.

With a setup on Elegant Breadboard (EBB) level (Fig. 1, left), frequency instabilities of $6 \times 10^{-15}$ at $1 \mathrm{~s}$ integration time and below $3 \times 10^{-15}$ for integration times between $100 \mathrm{~s}$ and 10,000 s have been demonstrated (Schuldt et al. 2017). This is, to our knowledge, the best published performance for such an iodine-based frequency reference. The frequency instabilities of the EBB setup-given in
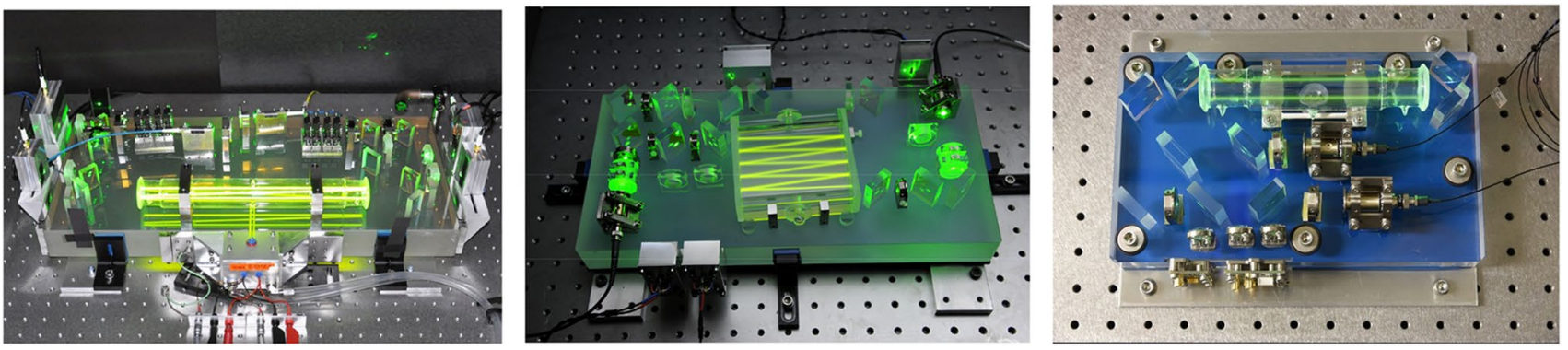

Fig. 1 Several implementations of the iodine spectroscopy unit, realized at DLR in collaboration with the universities HU Berlin and Bremen. Left: Elegant Breadboard (EBB, $25 \mathrm{~cm} \times 55 \mathrm{~cm})($ Schuldt et al. 2017); Middle: Engineering Model (EM, $18 \mathrm{~cm} \times 38 \mathrm{~cm})$
(Döringshoff et al. 2017); Right: Setup used on the sounding rocket mission JOKARUS $(15 \mathrm{~cm} \times 25 \mathrm{~cm}$ ) (Schkolnik et al. 2017; Döringshoff et al. 2019) 
Fig. 2 Measured frequency stability of the Iodine-EBB reference, given in Allan deviation. Shown is a beat measurement with a cavity setup (Schuldt et al. 2017), together with the frequency stabilities of the current Galileo clocks (RAFS, PHM) and the active hydrogen maser for ACES (SHM). Also shown is a beat measurement with a hydrogen maser (first measurement, not yet optimized, the degradation $>1000 \mathrm{~s}$ is believed to be an artefact). The measurement is limited by the H-maser performance for integration times $<1000 \mathrm{~s}$

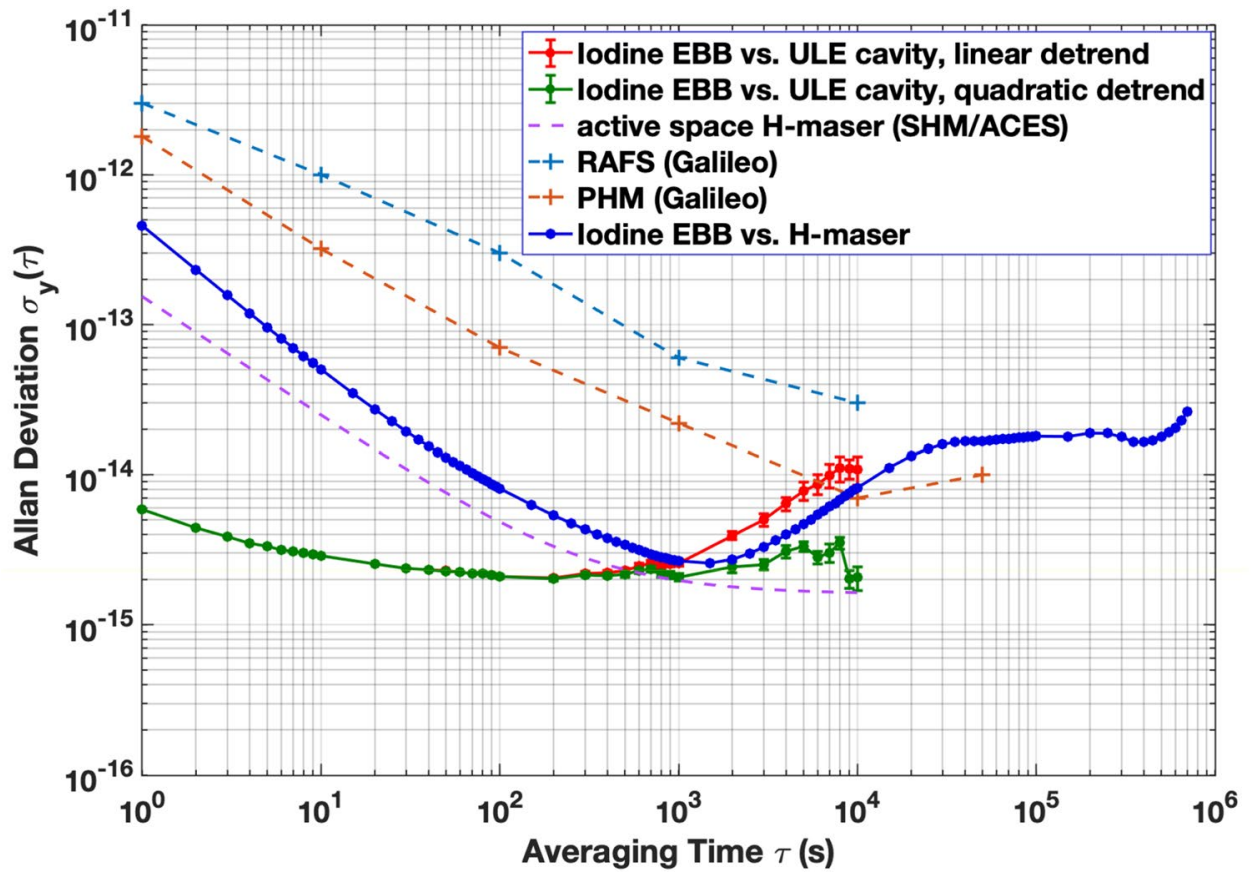

Allan deviation-are shown in Fig. 2, evaluated from a beat measurement with an ultra-low expansion (ULE) cavity setup. A linear and a second-order polynomial drift have been removed from the corresponding time records where the linear drift is attributed to the iso-thermal creep of the cavity. The Allan deviation shows white frequency noise for integration times between 1 and $10 \mathrm{~s}$ and Flicker noise of about $3 \times 10^{-15}$ at integration times $>1000 \mathrm{~s}$. For integration times up to $1000 \mathrm{~s}$, the frequency instabilities are 1-2 orders of magnitude lower than the one of the current Galileo clocks, i.e., RAFS and PHM. At longer integration times, the iodine reference approaches the performance of the Space Hydrogen Maser (SHM), an active hydrogen maser which is currently implemented within the ACES (Atomic Clock Ensemble in Space) mission on the ISS (Goujon et al. 2010). As our measurement of the iodine performance is most probably limited by the cavity reference, we started an investigation of the long-term stability of the EBB setup which is currently ongoing. A first frequency stability evaluation where the iodine EBB is compared to a hydrogen maser via an optical frequency comb is shown in Fig. 2 (blue curve). It is based on a 16 days continuous operation of the iodine reference.

The engineering model (EM) setup was further developed with respect to compactness and uses a specifically designed compact iodine cell, see Fig. 1, middle (Döringshoff et al. 2017). The EM spectroscopy unit was subjected to thermal cycling from $-20{ }^{\circ} \mathrm{C}$ to $+60{ }^{\circ} \mathrm{C}$ and vibrational loads with sine vibration up to $30 \mathrm{~g}$ and random vibration up to $25.1 \mathrm{~g}_{\mathrm{rms}}$. The frequency stability was measured before and after the tests where no degradation was observed. The frequency offset between the EBB and EM setups is below $1.5 \mathrm{kHz}$ with a reproducibility below $250 \mathrm{~Hz}$ (Döringshoff et al. 2017). While gas cells typically use a cold finger for setting the pressure inside the cell, the EM setup was also operated with a gas cell filled at an unsaturated vapor pressure of about $1 \mathrm{~Pa}$, showing similar performance. This allows lowering the complexity of the setup by omitting a temperature control and reducing the SWaP budgets.

An iodine-based frequency reference has been successfully operated on a sounding rocket (Schkolnik et al. 2017; Döringshoff et al. 2019) as part of the JOKARUS mission, showing autonomous operation during the $6 \mathrm{~min}$ long space flight. The setup was optimized with respect to the specific sounding rocket requirements, especially regarding dimensions. The compact spectroscopy unit with a $15 \mathrm{~cm}$ long iodine cell, is shown in Fig. 1, right, using a microintegrated extended cavity diode laser (ECDL) including a semiconductor power amplifier as light source (Kürbis et al. 2020). A short-term instability of the iodine reference of $1.5 \times 10^{-13} / \sqrt{ } \tau$ has been demonstrated (Döringshoff et al. 2019), probably limited due to its very compact design, intermodulation noise and the lack of intensity and RAM stabilization.

Within the ongoing project ADVANTAGE (Advanced Technologies for Navigation and Geodesy), a next iteration of the iodine setup is developed, based on the EBB-, EMand JOKARUS developments. The next step toward space instrumentation is carried out by a system level design where all components for the laser system, and the spectroscopy are integrated within one physical box. For the design, an absolute temperature of $15{ }^{\circ} \mathrm{C}$ with a stability of $\pm 5^{\circ} \mathrm{C}$ is 
assumed for the spacecraft interface plate. An actively controlled thermal shield within the iodine reference guarantees an operation of the spectroscopy board at $(22 \pm 0.1)^{\circ} \mathrm{C}$. The laser is fiber-coupled to this unit in the current design, where a Nd:YAG solid state laser is assumed baseline. In a design upgrade, a compact ECDL module will be integrated within the unit. Figure 3 shows a photograph of the integrated spectroscopy board, the corresponding optical layout for modulation transfer spectroscopy and a CAD model of the overall system level design of the reference.

The design of the ADVANTAGE setup is taken as basis for an optical reference developed for future GNSS. The corresponding schematic is shown in Fig. 4 where the frequency reference is split into two functional units: the iodine spectroscopy unit (including laser, fiber-optic components for beam preparation and frequency doubling) and the iodine control electronics (including laser driver, temperature controllers, AOM and EOM drivers, servo control loops for intensity, RAM and frequency stabilization). The stable light at a wavelength of $1064 \mathrm{~nm}$ is input to an optical frequency comb, which delivers a stable $10 \mathrm{MHz}$ clock signal.

A component-level breakdown, based on the design shown in Fig. 4 results in an overall mass of $20.1 \mathrm{~kg}$ (including a $20 \%$ component level margin), a power consumption of $43.1 \mathrm{~W}$ (including a 10\% component level margin) and a volume of 32.31 (without margin) of the optical reference (without frequency comb).

\section{In-orbit verification mission}

As part of a general technology development roadmap for future Galileo, DLR plans an in-orbit verification mission, called COMPASSO. This mission will demonstrate optical
Fig. 3 ADVANTAGE setup of the iodine reference. Top: Photograph and schematic of the spectroscopy board using a $22 \mathrm{~cm}$ long iodine cell in 4-pass configuration. Bottom: CAD at system level, showing the spectroscopy board within a thermal shield. The fiber-optic components for the laser system (including AOM and EOM) are mounted to the bottom side of the thermal shield, the SHG modules at one of its side plates. The laser is fiber coupled to this unit
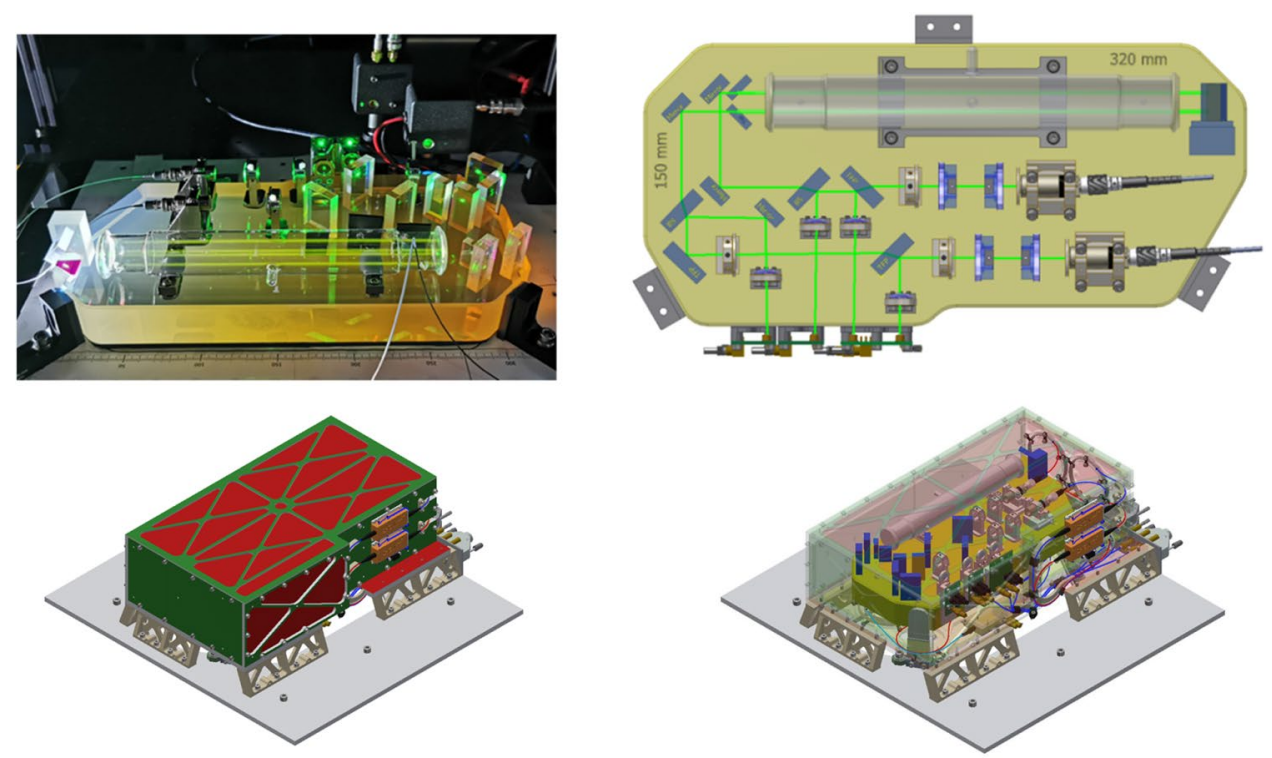

Fig. 4 Layout of the iodine reference with interfaces to the frequency comb, which delivers the stable $10 \mathrm{MHz}$ output signal. $E C D L$ extended cavity diode laser, $A O M$ acoustooptical modulator, EOM electrooptics phase modulator, $S H G$ second harmonic generation, $V H B G$ volume holographic bragg grating, $R A M$ residual amplitude modulation, $N C$ noise-canceling photo-detector, $P D$ photo-detector, $D D S$ direct digital synthesizer, $M O$ master oscillator, $P A$ power amplifier, $P I D$ proportional-integralderivative servo control, TM/TC telemetry and telecommand

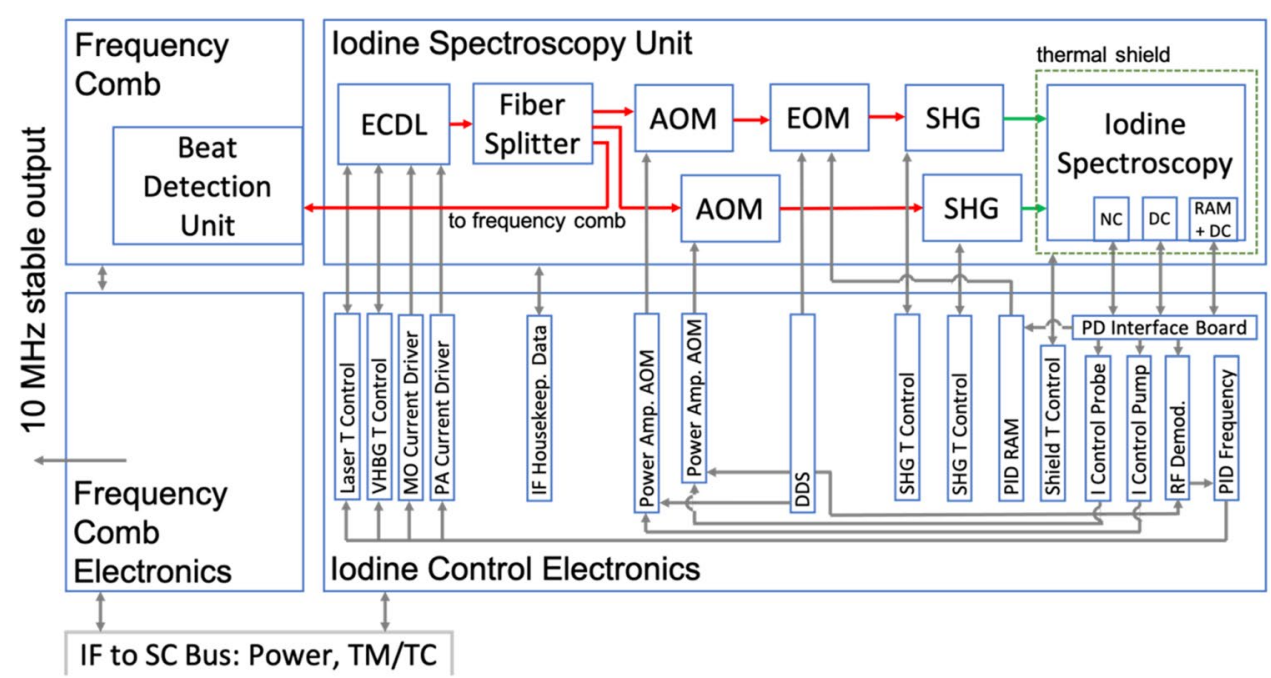


Fig. 5 High-level architecture of the COMPASSO payload

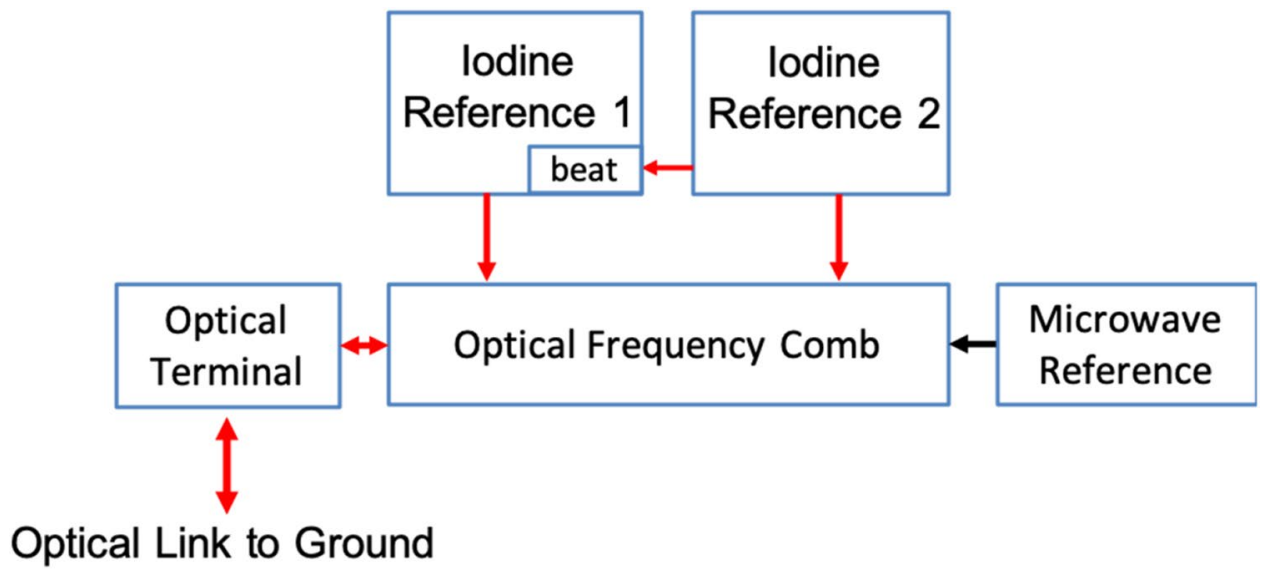

clock and optical link technologies on the Bartolomeo platform (Steimle et al. 2019) which is externally attached to the Columbus module of the ISS. Based on the mission concept presented by Schuldt et al. (2019), the mission feasibility of COMPASSO is currently investigated within a Phase A study at DLR where a mission lifetime of 2 years is assumed.

The payload consists of two iodine-based frequency references as detailed above, together with an optical frequency comb, a microwave frequency reference and an optical laser communication and ranging terminal, cf. the architecture shown in Fig. 5. The optical link is used for time- and frequency transfer and synchronization of a ground-based (microwave or optical) frequency reference to the space-based optical frequency references. Furthermore, it enables data communication and high-accuracy ranging.

The two iodine-based frequency references can be stabilized to the same or to different (nearby) ro-vibronic transitions. Their frequency stabilities are evaluated by comparing both references in the optical frequency range, i.e., near $282 \mathrm{THz}$ (corresponding to a wavelength of $1064 \mathrm{~nm}$ ). The optical frequency comb can be referenced to the iodine reference and transfers its frequency stability from the optical frequency range to the radio frequency range. Furthermore, the frequency comb can be referenced to an onboard microwave reference (e.g., a Galileo PHM or RAFS) and thus enables multiple comparison measurements with which the frequency stability in the relevant time period of the references can be evaluated. Using the two-way optical laser communication and ranging terminal (LCRT) the performance of the optical references onboard the ISS can additionally be compared to ground-based clocks.

Acknowledgements This work is supported by the Helmholtz-Gemeinschaft Deutscher Forschungszentren e.V. under grant number ZT-0007 (ADVANTAGE, Advanced Technologies for Navigation and Geodesy) and by the German Space Agency DLR with funds provided by the Federal Ministry of Economic Affairs and Energy (BMWi) under grant numbers 50QT1102, 50QT1201, 50WM1646 and 50NA1905. The iodine reference is investigated within the "Iodine Optical Clock
(IOC)" project under lead of SpaceTech GmbH, Immenstaad, Germany, with funding by the EU Horizon 2020 program.

Funding Open Access funding enabled and organized by Projekt DEAL.

Open Access This article is licensed under a Creative Commons Attribution 4.0 International License, which permits use, sharing, adaptation, distribution and reproduction in any medium or format, as long as you give appropriate credit to the original author(s) and the source, provide a link to the Creative Commons licence, and indicate if changes were made. The images or other third party material in this article are included in the article's Creative Commons licence, unless indicated otherwise in a credit line to the material. If material is not included in the article's Creative Commons licence and your intended use is not permitted by statutory regulation or exceeds the permitted use, you will need to obtain permission directly from the copyright holder. To view a copy of this licence, visit http://creativecommons.org/licenses/by/4.0/.

\section{References}

Argence B, Halloin H, Jeannin O, Prat P, Turazza O, de Vismes E, Auger G, Plagnol E (2010) Molecular laser stabilization at low frequencies for the LISA mission. Phys Rev D 81:082002. https://doi.org/10.1103/PhysRevD.81.082002

Barbarat J, Gillot J, Alvarez-Martinez H et al (2018) Compact and Transportable Iodine Frequency-Stabilized Laser. In: Proc SPIE 11180, International Conference on Space Optics, ICSO 2018, 111800T. https://doi.org/10.1117/12.2535948

Bongs K, Sing Y, Smith L et al (2015) Development of a strontium optical lattice clock for the SOC mission on the ISS. C R Phys 16:553-564. https://doi.org/10.1016/j.crhy.2015.03.009

Brewer AM, Chen JS, Hankin AM, Clements ER, Chou CW, Wineland DJ, Hume DB, Leibrandt DR (2019) ${ }^{27} \mathrm{Al}+$ Quantum-logic clock with a systematic uncertainty below $10^{-18}$. Phys Rev Lett 123:033201. https://doi.org/10.1103/PhysRevLett.123.033201

Cao J, Zhang P, Shang J, Cui K, Yuan J, Chao S, Wang S, Shu H, Huang X (2017) A compact, transportable single-ion optical clock with $7.8 \times 10^{-17}$ systematic uncertainty. Appl Phys B 123:112. https://doi.org/10.1007/s00340-017-6671-5

Chang P, Zhang S, Shang H, Chen J (2019) Stabilizing didoe laser to $1 \mathrm{~Hz}$-level Allan deviation with atomic spectroscopy for $\mathrm{Rb}$ four-level active optical frequency standard. Appl Phys B 125:196. https://doi.org/10.1007/s00340-019-7313-x 
Delehay M, Lacroute C (2018) Single-ion, transportable optical atomic clocks. J Mod Opt 65(5-6):622-639. https://doi.org/10. 1080/09500340.2018.1441917

Döringshoff K, Schuldt T, Kovalchuk E, Stühler J, Braxmaier C, Peters A (2017) A flight-like absolute optical frequency reference based on iodine for laser systems at $1064 \mathrm{~nm}$. Appl Phys B 123:183. https://doi.org/10.1007/s00340-017-6756-1

Döringshoff K et al (2019) Iodine frequency reference on a sounding rocket. Phys Rev Appl 11:054068. https://doi.org/10.1103/ PhysRevApplied.11.054068

Droz F, et al. (2006) The onboard Galileo clocks: Rubidium standard and Passive Hydrogen Maser-current status and performance. Proceedings of the 20th European Frequency and Time Forum, pp. 420-426.

Giorgi $\mathrm{G}$ et al (2019) Advanced technologies for satellite navigation and geodesy. Adv Space Res 64(6):1256-1273. https://doi.org/10. 1016/j.asr.2019.06.010

Goujon D et al (2010) Development of the space active hydrogen maser for the ACES mission. In: Proceedings of the 24th European Frequency and Time Forum, Noordwijk, pp 1-6. https://doi.org/10. 1109/EFTF.2010.6533730

Gutsch et al (2019) Towards a strontium beam optical reference based on the ${ }^{1} \mathrm{~S}_{0}{ }^{3} \mathrm{P}_{1}$ intercombination line on a sounding rocket. In: 2019 Joint Conference of the IEEE international frequency control symposium and european frequency and time forum (EFTF/IFC), Orlando. https://doi.org/10.1109/FCS.2019.8856031

Hannig $S$ et al (2019) Towards a transportable aluminium ion quantum logic optical clock. Rev Sci Instrum 90:053204. https://doi.org/ $10.1063 / 1.5090583$

Koller SB, Grotty J, Vogt S, Al-Masoudi A, Dörscher S, Häfner S, Sterr U, Lisdat C (2017) Transportable optical lattice clock with 7 x $10^{-17}$ uncertainty. Phys Rev Lett 118:073601. https://doi.org/ 10.1103/PhysRevLett.118.073601

Kürbis C, Bawamia A, Krüger M, Smol R, Peters A, Wicht A, Tränkle G (2020) Extended cavity diode laser master-oscillator-poweramplifier for operation of an iodine frequency reference on a sounding rocket. Appl Opt 50(2):253-262. https://doi.org/10. 1364/AO.379955

Leonhard V, Camp JB (2006) Space interferometry application of laser frequency stabilization with molecular iodine. Appl Opt 45(17):4142-4146. https://doi.org/10.1364/AO.45.004142

Lezius M et al (2016) Space-borne frequency comb metrology. Optica 3(12):1381-1387. https://doi.org/10.1364/OPTICA.3.001381

Martin KW et al (2018) Compact optical atomic clock based on a twophoton transition in rubidium. Phys Rev Appl 9:014019. https:// doi.org/10.1103/PhysRevApplied.9.014019

Maurice V et al (2020) Miniaturized optical frequency reference for next-generation portable optical clocks. Opt Express 28(17):24708-24720. https://doi.org/10.1364/OE.396296

McGrew WF et al (2018) Atomic clock performance enabling geodesy below the centimetre level. Nature 564:87-90. https://doi.org/10. 1038/s41586-018-0738-2

Newman ZL et al (2019) Architecture for the photonic integration of an optical atomic clock. Optica 6(5):680-685. https://doi.org/10. 1364/OPTICA.6.000680

Nicklaus K et al (2017) High stability laser for next generation gravity missions. In: Proc SPIE 10563, International Conference on Space Optics, ICSO 2014, 105632T. https://doi.org/10.1117/12.2304161

Nyholm K, Merimaa M, Ahola T, Lassila A (2003) Frequency Stabilization of a Diode-Pumped Nd: Yag Laser at $532 \mathrm{~nm}$ to Iodine by using third-harmonic technique. IEEE Trans Instrum Meas 52(2):284-287. https://doi.org/10.1109/TIM.2003.811679

Origlia $S$ et al (2018) Towards an optical clock for space: Compact, high-performance optical lattice clock based on bosonic atoms. Phys Rev A 98:053443. https://doi.org/10.1103/PhysRevA.98. 053443

Pröbster BJ, Lezius M, Mandel O, Braxmaier C, Holzwarth R (2021) FOKUS II-space flight of a compact and vacuum compatible dual frequency comb system. Accepted for publication by JOSA B

Riehle F, Gill P, Arias F, Robertsson L (2018) The CIPM list of recommended frequency standard values: guidelines and procedures. Metrologia 55(2):188-200. https://doi.org/10.1088/1681-7575/ aaa302

Schkolnik V et al (2017) JOKARUS-design of a compact optical iodine frequency reference for a sounding rocket mission. EPJ Quantum Technol 4:9. https://doi.org/10.1140/epjqt/ s40507-017-0063-y

Schuldt $\mathrm{T}$ et al (2019) In-orbit-verification of optical clock technologies. In: Proc. 70th International Aeronautics Conference (IAC), IAC-19-A2.1.2

Schuldt T, Döringshoff K, Kovalchuk E, Keetman A, Pahl J, Peters A, Braxmaier C (2017) Development of a compact optical absolute frequency reference for space with $10^{-15}$ instability. Appl Opt 56(4):1101-1106. https://doi.org/10.1364/AO.56.001101

Schuldt T, Döringshoff K, Oswald M, Kovalchuk EV, Peters A, Braxmaier C (2019) Absolute laser frequency stabilization for LISA. Int J Mod Phys D 28:12. https://doi.org/10.1142/S021827181 8450025

Shang H, Zhang X, Zhang S, Pan D, Chen H, Chen J (2017) Miniaturized calcium beam optical frequency standard using fully-sealed vacuum tube with $10^{-15}$ instability. Opt Express 25(24):3045930467. https://doi.org/10.1364/OE.25.030459

Steimle C, Walz C, Fuchs C, Pedersen D, Lombardi CM (2019) Bartolomeo external platform entering into commercial service. In: Proc. 70th International Aeronautics Conference (IAC), IAC-19-B3.3

Suemasa A, Shimo-oku A, Nakagawa K, Musha M (2017) Developments of high frequency and intensity stabilized lasers for space gravitational wave detector DECIGO/B-DECIGO. CEAS Space J 9:485-491. https://doi.org/10.1007/s12567-017-0151-y

Takamoto M, Ushijima I, Ohmae N, Yahagi T, Kokado K, Shinkai H, Katori H (2020) Test of general relativity by a pair of transportable optical lattice clocks. Nat Photonics 14:411-415. https://doi. org/10.1038/s41566-020-0619-8

Ushijima I, Takamoto M, Das M, Ohkubo T, Katori H (2015) Cryogenic optical lattice clocks. Nat Photonics 9:185-189. https://doi. org/10.1038/nphoton.2015.5

Zang EJ, Cao JP, Li Y, Li CY, Deng YK, Gao CQ (2007) Realization of four-pass i2 absorption cell in 532-nm optical frequency standard. IEEE Trans Instrum Meas 56(2):673-676. https://doi.org/10.1109/ TIM.2007.890816

Zhang S et al (2017) Compact Rb optical frequency standard with $10^{-15}$ stability. Rev Sci Instrum 88:103106. https://doi.org/10.1063/1. 5006962

Publisher's Note Springer Nature remains neutral with regard to jurisdictional claims in published maps and institutional affiliations. 

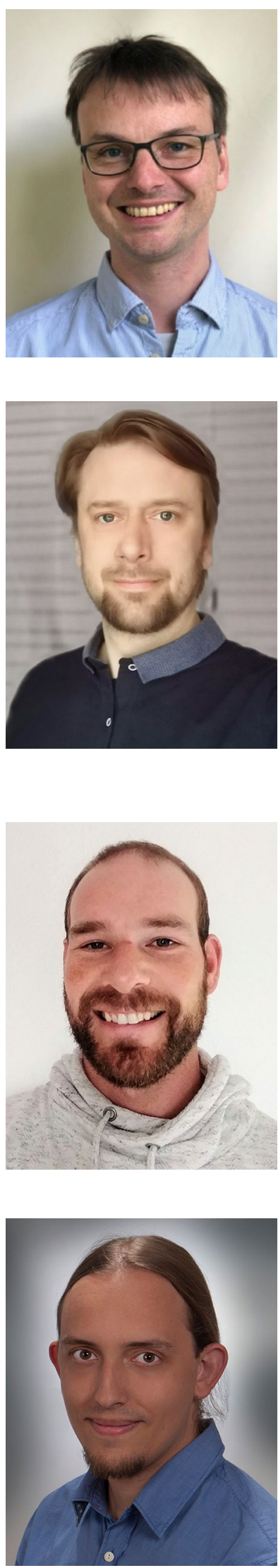

Thilo Schuldt joined the German Aerospace Center (DLR), Institute of Space Systems (Bremen) in 2013 and is group leader for "System Enabling Technologies". He received his Ph.D. from the Humboldt-Universität zu Berlin for his work on the space-based gravitational wave detector LISA. Thilo Schuldt has been working on many ESA and DLR funded projects in optical metrology, focusing on optical frequency references and highsensitivity laser interferometry.

Martin Gohlke joined the German Aerospace Center (DLR), Institute of Space Systems (Bremen) in 2012. He worked on the optical ground support equipment for the GRACE follow-on mission in cooperation with DLR institutes and space companies (Airbus, STI and JPL). LISA related topics such as laser interferometry, optical frequency references and temperature sensors are part of his current activities.

Markus Oswald is doing his $\mathrm{Ph}$.D. on iodine frequency references for space at the University of Bremen. After his master's degree from the HTWG Konstanz - University of Applied Sciences, in 2014, he worked on an FPGA-based digital laser locking system. Within the scope of his Ph.D. program, he has contributed to the development and realization of various iodine frequency reference setups for DLR and ZARM.

Jan Wüst joined the German Aerospace Center (DLR), Institute of Space Systems (Bremen) in 2019. He received his master's degree from the HTWG Konstanz - University of Applied Sciences. The thesis was about residual amplitude modulation in modulation transfer spectroscopy for frequency stabilized laser systems. Within the ADVANTAGE project, he is responsible for the mechanical and thermal design of the iodine spectroscopy subsystem.

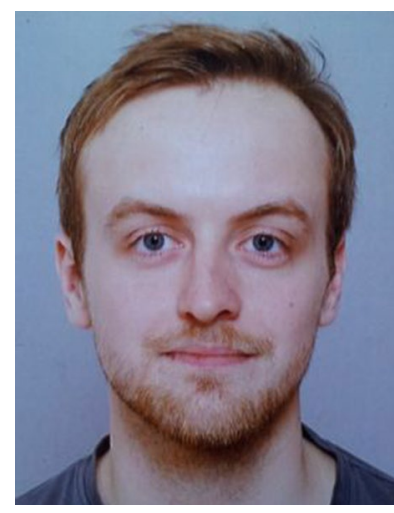

Tim Blomberg joined the German Aerospace Center (DLR), Institute of Space Systems (Bremen) in 2018. He received his master's degree from the RWTH Aachen University for the development, integration and test of an isostatic mounting for the iodine spectroscopy board of ADVANTAGE. Assembly, integration and test activities within ADVANTAGE are part of his current responsibilities.

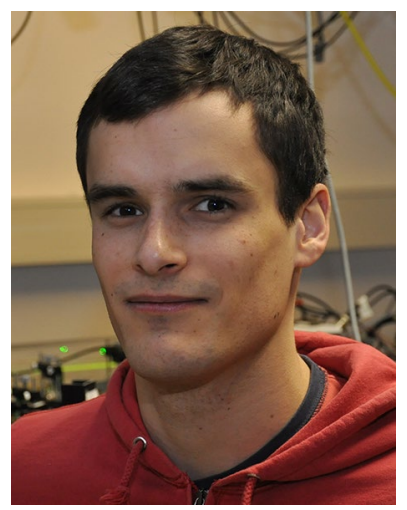

Klaus Döringshoff joined the Ferdinand-Braun-Institut, Leibniz-Institut für Höchstfrequenztechnik (Berlin) in 2020, and works on the development of optical frequency references based on high resolution laser spectroscopy. He received his Ph.D. from the Humboldt-Universität zu Berlin in 2018 for his work on the optical frequency references based on ro-virbronic transitions in molecular iodine.

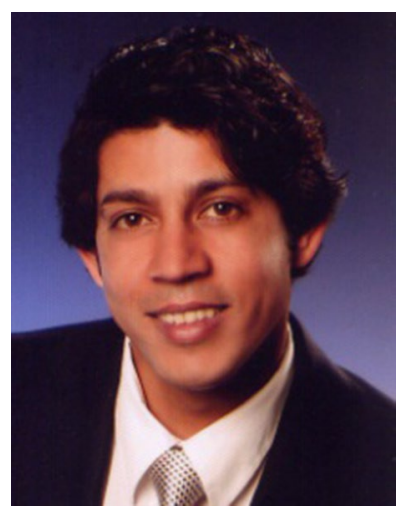

Ahmad Bawamia received a bachelor's degree in mathematics and physics at Université Bordeaux I in Bordeaux, France in 2001 and his master's in optoelectronics engineering at école nationale supérieure des sciences appliquées et de technologie in Lannion, France in 2004. He then joined the FerdinandBraun-Institut, Leibniz-Institut für Höchstfrequenztechnik $(\mathrm{FBH})$ in Berlin, Germany in 2005, working on the improvement of the spatial beam characteristics of broad-area semiconductor lasers. He obtained his doctoral degree at the technical university of Berlin in 2011. Since 2010, Ahmad is a full-time scientist at $\mathrm{FBH}$, where he specialized in the development of micro-integrated semiconductor laser modules for precision spectroscopy applications, especially in space. 


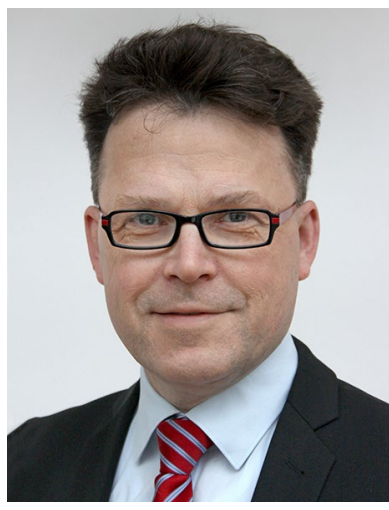

Andreas Wicht heads the Joint Lab Quantum Photonic Components at $\mathrm{FBH}$. He is an expert in the development of micro-integrated diode laser modules for applications that require stable lasers, including quantum optics with ultra-cold atoms. After receiving his $\mathrm{Ph} . \mathrm{D}$. from the University of Hannover, he joined Stanford University as a postdoc in 1999 to work on precision atom interferometers. Between 2002 and 2008 he was affiliated with the Universität of Düsseldorf as an assistant professor. In 2008 he joined the FBH to establish the Joint Lab Quantum Photonic Components, a Joint Lab between the FBH and the Humboldt-Universität zu Berlin.
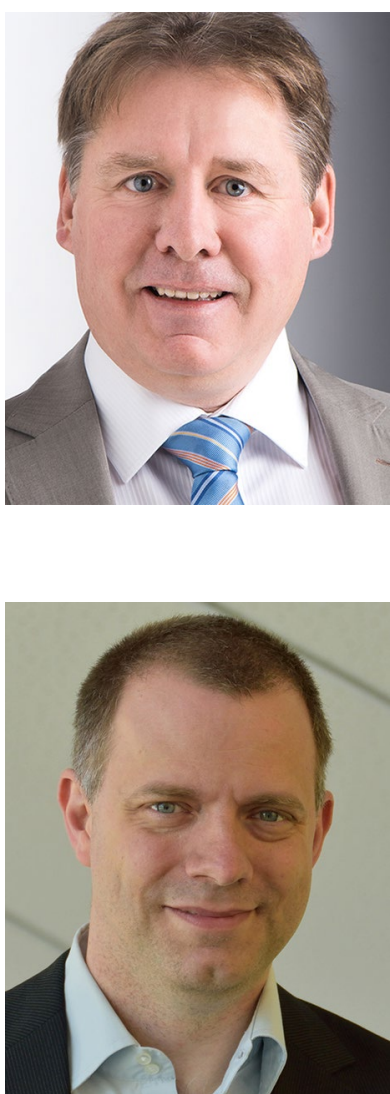

Matthias Lezius is group leader and senior scientist for "Space Combs" at Menlo Systems $\mathrm{GmbH}$ in Martinsried. $\mathrm{He}$ received his Ph.D. at the Innsbruck University in Austria for laser-interaction with rare-gas clusters. Since 2010 he has managed various DLR and ESA funded projects advancing the technology readiness, robustness and compactness of optical frequency combs. These combs have subsequently been used for scientific experiments on TEXUS sounding rockets.

Kai Voss is the head of optical instruments group at SpaceTech $\mathrm{GmbH}$ (STI). He received his Phd. in physics in 2004 and is working on laser-optical systems for space since 2011. He has been the system engineer at STI for the Laser ranging interferometer on GRACE FO, has led several technology studies and hardware projects.

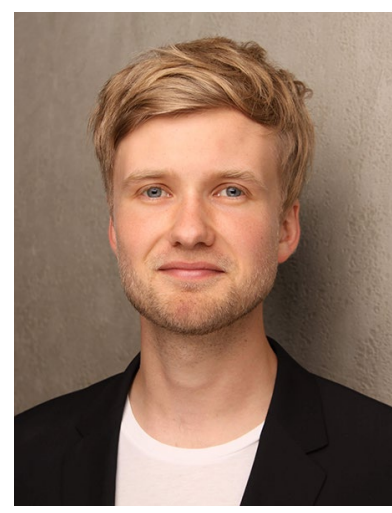

Markus Krutzik is head of the Joint Lab Integrated Quantum Sensors (IQS) operated by Ferdinand-Braun-Institut, LeibnizInstitut für Höchstfrequenztechnik (FBH) and the Humboldt-Universität zu Berlin. R\&D activities focus on the development of integrated atomic systems for frequency metrology, timing and field sensing applications. He got his Ph.D. in 2014 (HU Berlin), worked at the University of California, Berkeley, and NASA Jet Propulsion Laboratory,

Pasadena.

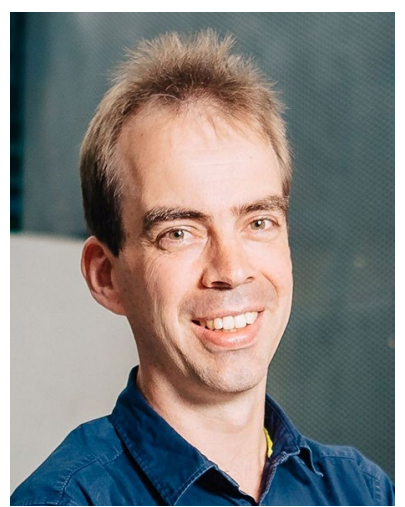

Sven Herrmann is a research scientist at the Center of Applied Space Technology and Microgravity at the University of Bremen. He received his Ph.D. from the Humboldt-Universität zu Berlin for his work on optical cavity-based frequency references for tests of fundamental physics. He has since contributed to several DLR and ESA funded activities in the field of fundamental physics, quantum sensing and cold atoms.

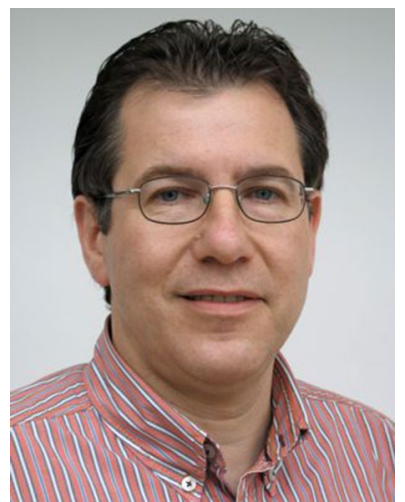

Evgeny Kovalchuk is a research scientist at the Humboldt-Universität zu Berlin, where he received his $\mathrm{Ph} . \mathrm{D}$. for his work on optical parametric oscillators for precision IR spectroscopy and metrology. His main research interests are laser stabilization, femtosecond combs, optical clock development, precision measurements, optical frequency metrology and phase noise measurements. 


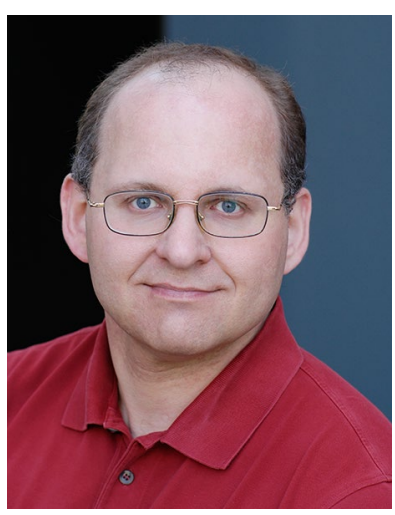

Achim Peters is professor for Optical Metrology at the physics department of the HumboldtUniversität zu Berlin. Since 2008 he is also affiliated with the Ferdinand-Braun-Institut, LeibnizInstitut für Höchstfrequenztechnik $(\mathrm{FBH})$ and the Joint Lab Quantum Photonic Components. He obtained his Ph.D. in physics at Stanford University in 1998 and has since then worked on many projects pursuing a variety of precision measurements as well as advancing the related technologies.

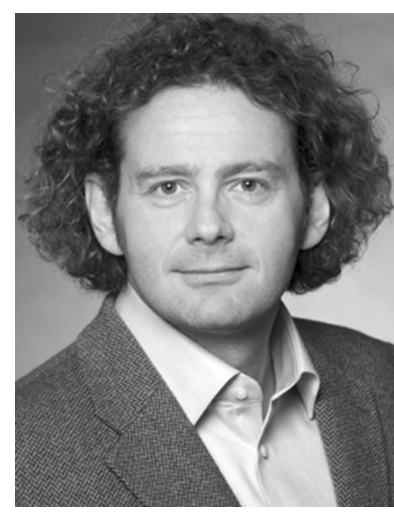

Claus Braxmaier is chair of Space Technology, University of Bremen, in cooperation with DLR. Braxmaier is head of the DLR-department "System Enabling Technologies" in Bremen. $\mathrm{He}$ received a $\mathrm{Ph}$.D. from the University of Konstanz and has been working on many ESA and DLR funded projects in space sciences, gravity missions, cold atom optics, quantum metrology, frequency references and laser interferometry. 